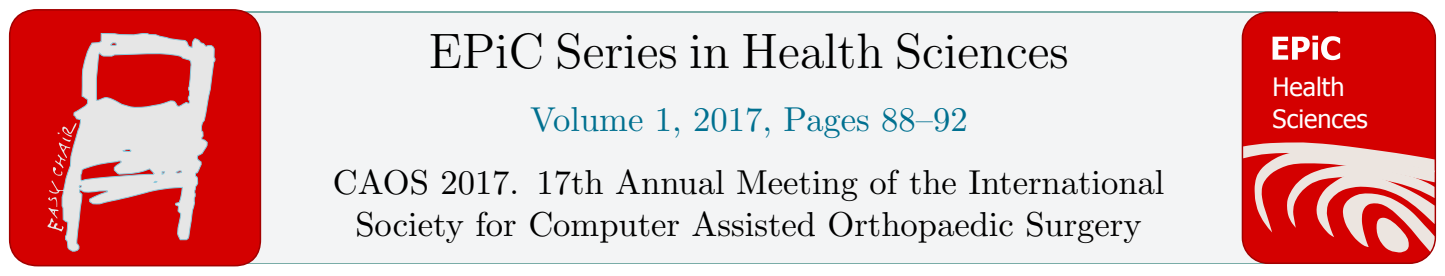

\title{
Relationship between pelvic morphology and functional parameters in standing position for patient-specific cup planning in THA
}

\author{
Maximilian C. M. Fischer ${ }^{1 *}$, Stephanie Schörner ${ }^{2}$, Stefan Rohde ${ }^{3}$, Christian \\ Lüring $^{2}$ and Klaus Radermacher ${ }^{1}$ \\ ${ }^{1}$ Chair of Medical Engineering, Helmholtz-Institute for Biomedical Engineering, RWTH Aachen \\ University, Aachen, Germany \\ ${ }^{2}$ Orthopädische Klinik, Klinikum Dortmund, Dortmund, Germany \\ ${ }^{3}$ Klinik für Radiologie und Neuroradiologie, Klinikum Dortmund, Dortmund, Germany \\ m.fischer@hia.rwth-aachen.de
}

\begin{abstract}
The sagittal orientation of the pelvis commonly called pelvic tilt has an effect on the orientation of the cup in total hip arthroplasty (THA). Pelvic tilt is different between individuals and changes during activities of daily living. In particular, the pelvic tilt in standing position should be considered during the planning of THA to adapt the target angles of the cup patient-specifically to minimize wear and the risk of dislocation. Methods to measure pelvic tilt require an additional step in the planning process, may be time consuming and additional devices or x-ray imaging are necessary.

In this study, the relationship between three functional parameters describing the sagittal pelvic orientation in standing position and seven morphological parameters of the pelvis was investigated. Correlations might be used to estimate the pelvic tilt in standing position by the morphology of the pelvis in order to avoid additional measuring techniques of pelvic tilt in the planning process of THA. For 18 subjects a semi-automatic process was established to match a 3D-reconstruction of the pelvis from CT scans to orthogonal EOS imaging in standing position and to calculate the morphological and functional parameters of the pelvis subsequently.

The two strongest correlations of the linear correlation analysis were observed between morphological pelvic incidence and functional sacral slope $(r=0.78 ; \mathrm{p}=$ 0.0001 ) and between morphological pubic symphysis-posterior superior iliac spinesratio and functional tilt of anterior pelvic plane $(r=-0.59 ; \mathrm{p}=0.0098)$. The results of this study suggest that patient-specific adjustments to the orientation of the cup in planning of THA without additional measurement of the sagittal pelvic orientation in standing position should be based on the correlation between morphological pelvic incidence and functional sacral slope.
\end{abstract}




\section{Introduction}

The sagittal orientation of the pelvis commonly called pelvic tilt has an effect on the orientation of the cup in total hip arthroplasty (THA). $10^{\circ}$ change of pelvic tilt changes anteversion from $7^{\circ}$ to $8^{\circ}$ and inclination around $3^{\circ}$ (Lembeck 2005; Babisch 2008; Tezuka 2015). Pelvic tilt is different between individuals and changes during activities of daily living. In particular the pelvic tilt in standing position should be considered during the planning of THA to adapt the target angles of the cup patient-specifically to minimize wear and the risk of dislocation (Maratt 2015). Navigation systems or customized jigs are used to transfer the results of the planning process based on supine CT images of the pelvic region to the surgical site. Different measuring techniques exist to determine the functional pelvic tilt in standing position (Dardenne 2009; Imai 2013; Murphy 2013). They all require an additional step in the planning process, may be time consuming and require additional devices or $\mathrm{x}$-ray imaging.

Different studies presented a correlation between the functional pelvic tilt in standing position and the morphological defined pelvic incidence (Eddine 2001; Vaz 2002; Loppini 2016). The results suggest that individual pelvic tilt has to be related to the morphology of the pelvis. In this study, the relationship between three functional parameters describing the sagittal pelvic orientation in standing position and seven morphological parameters of the pelvis was investigated. Correlations might be used to estimate the pelvic tilt in standing position by the morphology of the pelvis in order to avoid additional measuring techniques of pelvic tilt in the planning process of THA.

\section{Material and Methods}

18 patients of the orthopedics department, Klinikum Dortmund, Germany were retrospectively selected who underwent CT imaging of the pelvis and EOS imaging (Illes 2012) of the lower body for clinical purposes. A semi-automatic process was established to calculate the morphological and functional parameters of the pelvis. For each subject a surface model of the pelvis was created applying a threshold from $200 \mathrm{HU}$ to $1600 \mathrm{HU}$ to the CT volume (Sugano 2001). If necessary, the resulting volume was manually edited to separate the pelvis from the adjacent bones and close holes.

In a next step, morphological landmarks and parameters were determined applying several automatic methods to the mesh. The orientation of the surface model of the pelvis was unified using an approach of Kai et al. based on the automatic detection of the pubic symphysis (PS) and the anterior superior iliac spines (ASIS) (Kai 2014). The method was substantially extended and enhanced to identify the posterior superior iliac spines (PSIS), the anterior inferior iliac spines (AIIS) and the ischial spine (IS) in addition. The hip joint centers (HJC) where automatically detected using a method proposed by Cerveri et al. based on the curvature of the mesh (Cerveri 2011). In a similar manner, the sacral plateau was identified including the sacral promontory as well as the center of the sacral plateau (sacral center). Seven morphological parameters have been calculated subsequently:

- Pelvic incidence: Angle between the normal of the plane fitted to the sacral plateau (sacral plane) pointing in caudal direction and the plane defined by the sacral center and HJC.

- ASIS PSIS ratio: The middle between both HJC is projected on the line connecting the middles between both ASIS points and both PSIS points (ASIS PSIS line). The ASIS PSIS line is intersected in two parts by the projection and the ASIS PSIS ratio is defined as quotient of the length of both parts. The AIIS PSIS ratio, PS PSIS ratio, ASIS IS ratio, AIIS IS ratio and PS IS ratio was calculated in the same way.

In a next step, four landmarks were manually selected in the frontal and the lateral EOS images to register the surface model of the pelvis to the orthogonal EOS-imaging in standing position: pubic 
symphysis, sacral promontory and the two hip joint centers (Figure 1). After the rigid landmark-based registration, three functional parameters were calculated:

- Sacral slope: The angle between the horizontal plane and the sacral plane

- Sacral (pelvic) tilt: The angle between the vertical plane and the plane defined by the sacral center and $\mathrm{HJC}$

- APP (pelvic) tilt: The angle between the vertical plane and the anterior pelvic plane (APP) defined by PS and ASIS

The relationship between the functional and morphological parameters was evaluated using Pearson linear correlation coefficient (PCC).

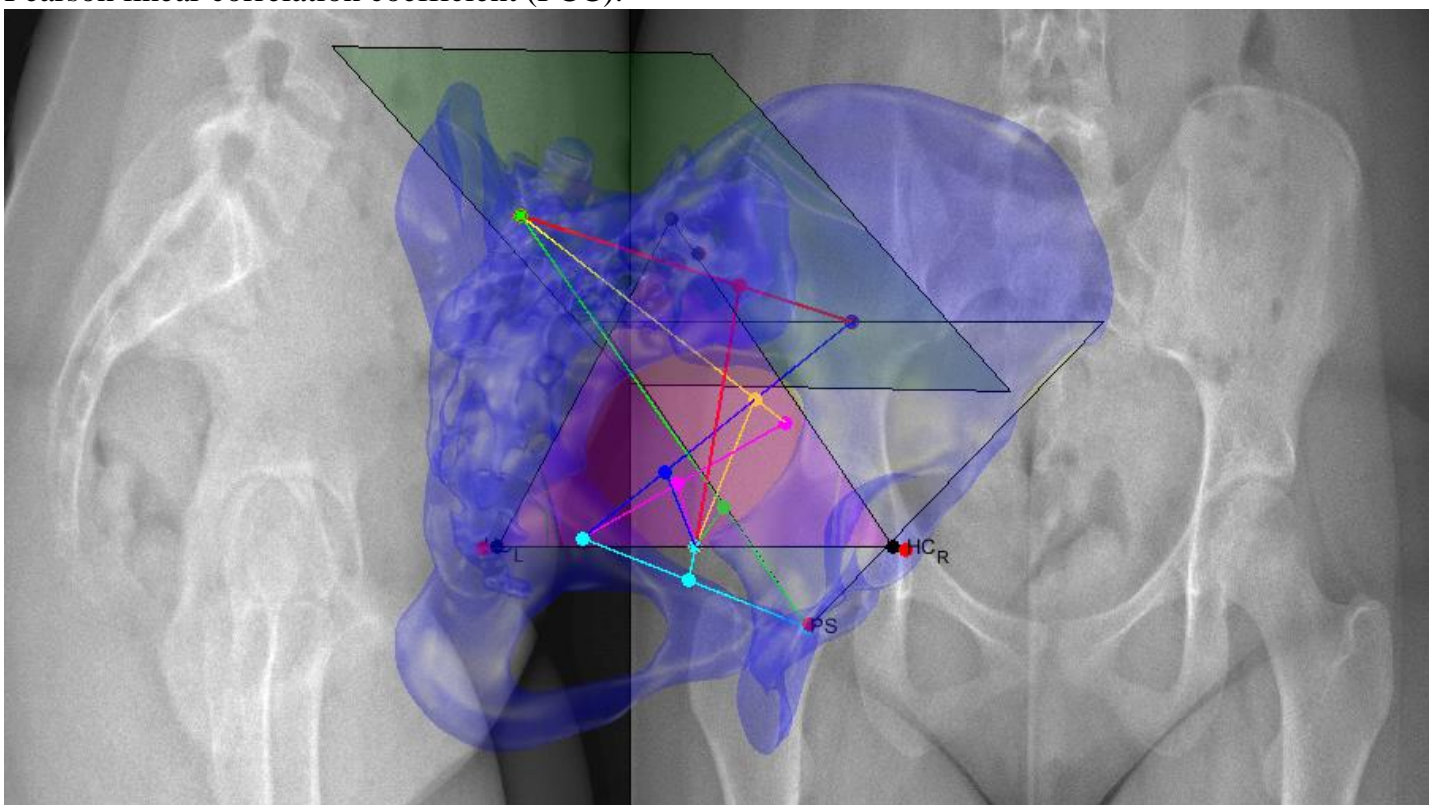

Figure 1: The model of the pelvis registered to the orthogonal EOS images in standing position. The sacral plane is depicted in green, the plane defined by the sacral center and HJC in magenta and the APP in yellow. The ASIS PSIS ratio is depicted in red, the AIIS PSIS ratio in yellow, the PS PSIS ratio in green, the ASIS IS ratio in blue, the AIIS IS ratio in magenta and the PS IS ratio in cyan.

\section{Results}

The results of the linear correlation analysis are presented in Table 1. For significance level of $\mathrm{p}<0.001$ the PCC between pelvic incidence and sacral slope is $0.78(\mathrm{p}=0.0001)$. For a significance of $\mathrm{p}<0.01$ the PCC between PS PSIS ratio and APP tilt is $-0.59(\mathrm{p}=0.0098)$. For a significance level of $\mathrm{p}<0.05$ PCC between pelvic incidence and functional sacral tilt is $0.51(\mathrm{p}=0.0316)$ and PCC between PS IS ratio and APP tilt is $-0.49(\mathrm{p}=0.0382)$. 


\begin{tabular}{|l|c|c|c|c|c|c|}
\hline \multicolumn{1}{|c|}{$\mathrm{n}=18$} & \multicolumn{6}{c|}{ Functional parameters } \\
\hline \multirow{2}{*}{$\begin{array}{c}\text { Morphological } \\
\text { parameters }\end{array}$} & \multicolumn{2}{|c|}{ APP tilt } & \multicolumn{2}{c|}{ Sacral tilt } & \multicolumn{2}{c|}{ Sacral slope } \\
\cline { 2 - 7 } & $\mathrm{r}$ & $\mathrm{p}$ & $\mathrm{r}$ & $\mathrm{p}$ & $\mathrm{r}$ & $\mathrm{p}$ \\
\hline Pelvic incidence & -0.26 & 0.2920 & 0.51 & 0.0316 & 0.78 & 0.0001 \\
\hline ASIS PSIS ratio & -0.06 & 0.8177 & 0.27 & 0.2734 & -0.21 & 0.4102 \\
\hline AIIS PSIS ratio & -0.12 & 0.6438 & -0.02 & 0.9223 & -0.47 & 0.0504 \\
\hline PS PSIS ratio & -0.59 & 0.0098 & 0.41 & 0.0939 & 0.01 & 0.9537 \\
\hline ASIS IS ratio & -0.25 & 0.3186 & 0.15 & 0.5424 & -0.17 & 0.4967 \\
\hline AIIS IS ratio & -0.28 & 0.2550 & -0.02 & 0.9465 & -0.32 & 0.2004 \\
\hline PS IS ratio & -0.49 & 0.0382 & 0.32 & 0.2016 & 0.11 & 0.6525 \\
\hline
\end{tabular}

Table 1: PCC r- and p-value between morphological and functional parameters. Correlations with $\mathrm{p}<0.001$ are shaded in dark grey, correlations with $\mathrm{p}<0.01$ in grey and correlations with $\mathrm{p}<0.05$ are shaded in light grey.

\section{Discussion}

The strongest correlation was observed between the pelvic incidence and sacral slope $(r=0.78$, $\mathrm{p}<0.001)$. Similar results have been reported by Eddine et al. $(r=0.82, p<0.05)$, Vaz et al. $(r=0.86$, $\mathrm{p}<0.001)$ and Loppini et al. $(\mathrm{r}=0.66, \mathrm{p}<0.0001)$ (Eddine 2001; Vaz 2002; Loppini 2016). By geometrical definition, the pelvic incidence equals the sum of sacral slope and sacral tilt. But correlation of pelvic incidence and sacral tilt $(\mathrm{r}=0.51, \mathrm{p}<0.05)$ is lower than the correlation of pelvic incidence and sacral slope. Eddine et al. $(r=0.73, \mathrm{p}<0.05)$, Vaz et al. $(r=0.59, \mathrm{p}<0.001)$ and Loppini et al. $(\mathrm{r}=0.65, \mathrm{p}<0.0001)$ observed the same tendency. In this study, the deviation may be explained by the low number of subjects. In agreement with literature pelvic incidence did not correlate with APP tilt, but in this study a correlation was found between PS PSIS ratio and APP tilt $(\mathrm{r}=-0.59$, $\mathrm{p}<0.01)$.

Further investigation should include more subjects to verify these relations. Beside the low number of subjects, other limitations have to be considered. All subjects are patients with hip or spine problems and some subjects have skeletal deformities. Subjects may have adapted the sagittal pelvic orientation to reduce pain. The automatic landmark detection worked robustly for all subjects, but has to be validated against the manual identification. The landmark-based registration should be replaced by an automatic $2 \mathrm{D}-3 \mathrm{D}$ registration method between the orthogonal EOS images and the model of the pelvic bone.

The results of this study suggest that patient-specific adjustments to the orientation of the cup in planning of THA without additional measurement of the sagittal pelvic orientation in standing position should be based on the correlation between morphological pelvic incidence and functional sacral slope. Further studies may consider body mass index of the subjects within a multi linear regression analysis.

\section{References}

Babisch JW, Layher F, Amiot L-P (2008): The rationale for tilt-adjusted acetabular cup navigation, The Journal of bone and joint surgery. American volume 90 (2), 357-365 
Cerveri P, Marchente M, Chemello C, Confalonieri N, Manzotti A, Baroni G (2011): Advanced computational framework for the automatic analysis of the acetabular morphology from the pelvic bone surface for hip arthroplasty applications, Annals of biomedical engineering 39 (11), 2791-2806

Dardenne G, Dusseau S, Hamitouche C, Lefevre C, Stindel E (2009): Toward a dynamic approach of THA planning based on ultrasound, Clinical orthopaedics and related research 467 (4), 901-908

Eddine TA, Migaud H, Chantelot C, Cotten A, Fontaine C, Duquennoy A (2001): Variations of pelvic anteversion in the lying and standing positions: analysis of 24 control subjects and implications for CT measurement of position of a prosthetic cup, Surgical and radiologic anatomy : SRA 23 (2), $105-110$

Illes T, Somoskeoy S (2012): The EOS imaging system and its uses in daily orthopaedic practice, International orthopaedics 36 (7), 1325-1331

Imai N, Ito T, Suda K, Miyasaka D, Endo N (2013): Pelvic flexion measurement from lateral projection radiographs is clinically reliable, Clinical orthopaedics and related research 471 (4), 12711276

Kai S, Sato T, Koga Y, Omori G, Kobayashi K, Sakamoto M, Tanabe Y (2014): Automatic construction of an anatomical coordinate system for three-dimensional bone models of the lower extremities--pelvis, femur, and tibia, Journal of biomechanics 47 (5), 1229-1233

Lembeck B, Mueller O, Reize P, Wuelker N (2005): Pelvic tilt makes acetabular cup navigation inaccurate, Acta orthopaedica 76 (4), 517-523

Loppini M, Longo UG, Ragucci P, Trenti N, Balzarini L, Grappiolo G (2016): Analysis of the Pelvic Functional Orientation in the Sagittal Plane: A Radiographic Study With EOS 2D/3D Technology. Accepted Manuscript, The Journal of arthroplasty

Maratt JD, Esposito CI, McLawhorn AS, Jerabek SA, Padgett DE, Mayman DJ (2015): Pelvic tilt in patients undergoing total hip arthroplasty: when does it matter?, The Journal of arthroplasty 30 (3), 387-391

Murphy WS, Klingenstein G, Murphy SB, Zheng G (2013): Pelvic tilt is minimally changed by total hip arthroplasty, Clinical orthopaedics and related research 471 (2), 417-421

Sugano N, Sasama T, Nakajima Y, Sato Y, Nishii T, Iida T, Nakagawa K, Ono K, Nishihara S, Tamura S, Yonenobu K, Ochi T (2001): Effects of CT threshold value to make a surface bone model on accuracy of shape-based registration in a CT-based navigation system for hip surgery, International Congress Series 1230 , 319-324

Tezuka T, Inaba Y, Kobayashi N, Ike H, Kubota S, Kawamura M, Saito T (2015): Influence of Pelvic Tilt on Polyethylene Wear after Total Hip Arthroplasty, BioMed research international 2015 , 327217

Vaz G, Roussouly P, Berthonnaud E, Dimnet J (2002): Sagittal morphology and equilibrium of pelvis and spine, European spine journal : official publication of the European Spine Society, the European Spinal Deformity Society, and the European Section of the Cervical Spine Research Society $11(1), 80-87$ 